\title{
AVALIAÇÃO DOS RESULTADOS DO TRATAMENTO CIRÚRGICO DA ESCOLIOSE NA ATROFIA MUSCULAR ESPINHAL TIPO 2
}

\author{
RESULTS EVALUATION OF SURGICAL TREATMENT OF SCOLIOSIS IN \\ SPINAL MUSCULAR ATROPHY TYPE 2 \\ EVALUACIÓN DE LOS RESULTADOS DEL TRATAMIENTO QUIRÚRGICO DE LA
ESCOLIOSIS EN LA ATROFIA MUSCULAR ESPINAL TIPO 2
}

Luiz Eduardo Munhoz da Rocha', Edson Pudles², Henrique Bonotto Lampert ${ }^{3}$

\begin{abstract}
RESUMO
Objetivo: Avaliar o resultado do tratamento cirúrgico da escoliose em pacientes com atrofia muscular espinhal (AME) tipo 2. Método: Estudo retrospectivo com 12 pacientes portadores de AME tipo 2 submetidos à artrodese e instrumentação para correção da escoliose com mais de dois anos de seguimento. Foi avaliado o grau e percentual de correção da deformidade e da obliquidade pélvica no pós-operatório e a perda na última avaliação, além das complicações e o impacto do tratamento sobre a função respiratória. Resultados: 0 seguimento médio foi de 77,5 meses (6,4 anos) $\pm 58,9$ meses (4,9 anos), o ângulo de Cobb pré-operatório médio foi de $76,1^{\circ} \pm 31,7^{\circ}$ $\left(35^{\circ}\right.$ a $\left.144^{\circ}\right)$ e no pós-operatório de $29,5^{\circ} \pm 23,2^{\circ}\left(5^{\circ}\right.$ a $\left.90^{\circ}\right)$, com a correção média de $46,6^{\circ}(61,29 \%)$. A obliquidade pélvica média no pré-operatório foi de $15,1^{\circ} \pm 13,3^{\circ}$ (variação de $0^{\circ}$ a $37^{\circ}$ ), e no pós-operatório de $8,5^{\circ} \pm 9,9^{\circ}$ (variação de $0^{\circ}$ a $30^{\circ}$ ), com uma correção média de 6,5 $(43,37 \%)$. Cinco pacientes tiveram complicações (41,6\%). A Capacidade Ventilatória Forçada (CVF) média pré-operatória foi de $62,9 \% \pm 38,6 \%$ (variação de $23,3 \%$ a $89 \%$ ) e de $45,9 \% \pm 25,0 \%$ (variação de $15 \%$ a $86,2 \%$ ), na última avaliação. O declínio foi de $17 \%$ da capacidade vital, com redução de 2,4\% por ano de seguimento. Conclusões: O tratamento cirúrgico da escoliose em pacientes com AME permite corrigir a obliquidade pélvica e restabelecer o balanço sagital e coronal liberando as mãos para as atividades da vida diária. A função pulmonar foi afetada positivamente pelo tratamento.
\end{abstract}

Descritores: Atrofia muscular espinhal; Escoliose/cirurgia; Artrodese; Fusão vertebral; Testes de função respiratória.

\begin{abstract}
Objective: To evaluate the outcome of surgical treatment of scoliosis in patients with spinal muscular atrophy (SMA) type 2. Methods: A retrospective study with 12 patients with SMA type 2 who underwent arthrodesis and instrumentation for scoliosis correction with more than two years of follow-up. The degree and rate of correction of deformity and pelvic obliquity postoperatively and loss in the last evaluation were evaluated, in addition to the complications and the impact of treatment on respiratory function. Results: Mean follow-up was 77.5 months (6.4 years) \pm 58.9 months (4.9 years), Cobb angle before surgery averaged $76.1^{\circ} \pm 31.7^{\circ}\left(35^{\circ}\right.$ to $144^{\circ}$ ) and postoperatively was $29.5^{\circ} \pm$ $23.2^{\circ}\left(5^{\circ}\right.$ to $\left.90^{\circ}\right)$, with correction average of $46.6^{\circ}(61.29 \%)$. The average pelvic obliquity preoperatively was $15.1^{\circ} \pm 13.3^{\circ}$ (range $0^{\circ}$ to $37^{\circ}$, and $8.5^{\circ} \pm 9.9^{\circ}$ (range $0^{\circ}$ to $30^{\circ}$ ) postoperatively, with an average correction of $6.5^{\circ}(43.37 \%)$. Five patients had complications (41.6\%). The forced ventilatory capacity (FVC) preoperative average was $62.9 \% \pm 38.6 \%$ (range $23.3 \%$ to $89 \%$ ) and $45.9 \% \pm 25.0 \%$ (range $15 \%$ to $86.2 \%$ ) in the last evaluation. The decline was $17 \%$ of vital capacity, with a reduction of $2.4 \%$ per year of follow-up. Conclusions: Surgical treatment of scoliosis in patients with SMA allows the correction of pelvic obliquity and restoration of the sagittal and coronal balance freeing the hands for activities of daily living. Lung function was positively affected by treatment.
\end{abstract}

Keywords: Muscular atrophy, spinal; Scoliosis/surgery; Arthrodesis; Spinal fusion; Respiratory function tests.

\section{RESUMEN}

Objetivo: Evaluar los resultados del tratamiento quirúrgico de la escoliosis en pacientes con atrofia muscular espinal (AME) de tipo 2. Métodos: Estudio retrospectivo de 12 pacientes con atrofia muscular espinal tipo 2 que fueron sometidos a artrodesis e instrumentación para la corrección de la escoliosis, con más de dos años de seguimiento. En la última evaluación, se evaluaron el grado y el porcentaje de corrección de la deformidad y la oblicuidad pélvica después de la operación y la pérdida, además de las complicaciones y el impacto del tratamiento sobre la función respiratoria. Resultados: El promedio de seguimiento fue 77,5 meses (6,4 años) \pm 58,9 meses (4,9 años), el ángulo de Cobb antes de la cirugía en promedio $76,1^{\circ} \pm 31,7^{\circ}\left(35^{\circ}\right.$ a $\left.144^{\circ}\right)$ y el postoperatorio fue $29,5^{\circ} \pm 23,2^{\circ}\left(5^{\circ}\right.$ a $\left.90^{\circ}\right)$, con un promedio de corrección de $46,6^{\circ}(61,29 \%)$. La oblicuidad pélvica promedio en el preoperatorio fue $15,1^{\circ} \pm 13,3^{\circ}$ (variación de $0^{\circ}$ a $37^{\circ}$ ) y después de la operación $8,5^{\circ} \pm 9,9^{\circ}$ (variación de $0^{\circ}$ a $30^{\circ}$ ), con una corrección promedio de $6,5^{\circ}(43,37 \%)$. Cinco pacientes presentaron complicaciones (41,6\%). La Capacidad Ventilatoria Forzada (CVF) preoperatoria promedio fue 62,9\% \pm 38,6\% (variación de 23,3\% a 89\%), y 45,9\% $\pm 25,0 \%$ (variación de 15\% a 86,2\%) en la última evaluación. La disminución fue de 17\% de la capacidad vital, con una reducción de 2,4\% por año de seguimiento. Conclusiones: El tratamiento quirúrgico de la escoliosis, en pacientes con AME, permite la corrección de la oblicuidad pélvica y restaurar el equilibrio sagital y coronal, liberando las manos para las actividades de la vida diaria. La función pulmonar se vio afectada positivamente por el tratamiento.

Descriptores: Atrofia muscular espinal; Escoliosis/cirugía; Artrodesis; Fusión vertebral; Pruebas de función respiratoria.

1 - Médico, Preceptor da Cirurgia da Coluna do Serviço de Ortopedia eTraumatologia do Hospital Pequeno Príncipe de Curitiba - HPP. Curitiba. PR. Brasil.

2 - Médico, Preceptor da Cirurgia da Coluna do Serviço de Ortopedia eTraumatologia do Hospital Universitário Evangélico de Curitiba - HUEC. Curitiba. PR. Brasil.

3 - Médico Estagiário do Grupo de Cirurgia da Coluna do Departamento Ortopedia eTraumatologia do Hospital Pequeno Príncipe de Curitiba. HPP. Curitiba. PR. Brasil.

Trabalho realizado no Serviço de Ortopedia eTraumatologia do Hospital Pequeno Príncipe de Curitiba. PR. Brasil.

Correspondência: Henrique Bonotto Lampert. Rua Alf Poli, 464. CEP 80730-000 - Curitiba. PR. Brasil. Email: hblampert@ hotmail.com 


\section{INTRODUÇÃO}

A atrofia muscular espinhal (AME) é doença genética de herança autossômica recessiva que afeta o corpo do neurônio motor no corno anterior da medula espinhal e tem a prevalência de 8:100,000 nascidos vivos. É a doença neuromuscular fatal mais comum da infância e o terceiro diagnóstico de doença neuromuscular mais freqüente em crianças abaixo dos 18 anos $^{1-3}$.

Conforme a época do início das manifestações clínicas doença e a gravidade do comprometimento motor, a AME é classificada por Byers e Baker em três formas: ${ }^{4,5}$

A infantil aguda (doença de Werdnig-Hoffmann aguda ou atrofia muscular espinhal tipo I) é diagnosticada nos primeiros meses de vida. Geralmente estes pacientes raramente sobrevivem após os dois anos de idade, devido à insuficiência respiratória. A doença se caracteriza pela ausência de movimentos espontâneos das extremidades, arreflexia, fasciculação de língua e por tremores finos dos dedos. A criança assume a postura de batráquio dos membros inferiores, a função da musculatura intercostal é débil e a respiração é diafragmática. Essas crianças interagem apropriadamente para sua idade.

$\mathrm{Na}$ atrofia muscular espinhal tipo II (doença de Werdnig-Hoffmann crônica) as crianças são hipotônicas e tem o desenvolvimento motor normal até cerca de seis a oito meses de idade. O envolvimento dos membros inferiores é mais evidente, conseguem sentar com apoio, mas raramente deambular. A sobrevida é variável, sendo que muitos pacientes atingem a terceira e quarta década de vida.

A atrofia muscular espinhal tipo III (doença de KugelbergWelander) se apresenta com um quadro clínico mais brando, manifestando-se na infância tardia ou adolescência. Estes pacientes geralmente são deambuladores, mas com sinal de trendelemburg e hiperlordose lombar devido à insuficiência da musculatura antigravitária. Com o balanço sagital negativo o gasto de energia da marcha é menor e permite a bipedestação por mais tempo. A habilidade qualidade da deambulação está relacionada a idade do início da manifestação da doença².

A AME apresenta herança autossômica recessiva de distribuição quase igual entre os sexos, com leve predominância no sexo masculino ${ }^{1}$. Mais de $98 \%$ dos casos são causados por mutações na porção telomérica do gene SMN, sendo a análise deste de grande valor para o diagnóstico?

A escoliose é a principal manifestação ortopédica da doença, a sua prevalência e magnitude estão diretamente relacionadas a capacidade de deambulação e a gravidade da doença. Sua apresentação típica é uma curva longa, em "C", dextroconvexa, associadas à obliquidade pélvica e $90 \%$ curvas únicas. Destas, $80 \%$ são toracolombares e $20 \%$ torácicas. Inicialmente as curvas flexíveis apesar do grande valor angular e evoluem com rápida progressão e piora da capacidade vital. A história natural da escoliose não tratada inclui: perda da capacidade sentar e de usar os membros superiores devido à necessidade de usá-los para suporte do tronco; bursite isquiática com escara de decúbito; dor lombar, impacto da coluna contra a crista ilíaca e comprometimento cardiopulmonar progressivo secundário a doença restritiva pulmonar ${ }^{5}$.

As indicações de tratamento cirúrgico são: deformidade progressiva refratária ao tratamento ortótico e curvas maiores de 40 a 60 graus e perda da capacidade de sentar. $O$ tratamento geralmente consiste da artrodese com instrumentação de T2 ao sacro por acesso posterior, alguns autores tem utilizado as hastes de crescimento (dual growing rod) nas crianças imaturas. $O$ acesso anterior é eventualmente indicado em curvas de grande magnitude (>100 graus), em pacientes muito imaturos e sem comprometimento pulmonar, embora muitos autores não o recomendem, devido ao impacto negativo na capacidade vital ${ }^{5}$.

As complicações pós-operatórias relacionadas ao tratamento cirúrgico da escoliose é frequente, variando $24-75 \%$. Sendo as principais as pulmonares e infecciosas ${ }^{6}$

Deterioração da função pulmonar é a principal causa da mortalidade em pacientes com doenças neuromusculares, como a $\mathrm{AME}^{7}$. A melhora da função pulmonar, após o tratamento cirúrgico, apresenta resultados controversos na literatura. Alguns estudos demonstram deterioração continua no pósoperatório, outros não demonstraram qualquer modificação e outro observou melhora da função pulmonar. Apesar dos resultados relatados o impacto positivo do tratamento cirúrgico da escoliose na função pulmonar destes pacientes não está bem estabelecido ${ }^{8}$.

O objetivo deste estudo retrospectivo é avaliar os resultados da correção cirúrgica da escoliose nos pacientes portadores de AME tipo 2 comparando os estudos radiográficos pré, pós-operatória e na ultima consulta, a função pulmonar, bem como a complicações e ganhos relacionados ao tratamento (Figuras 1-5).

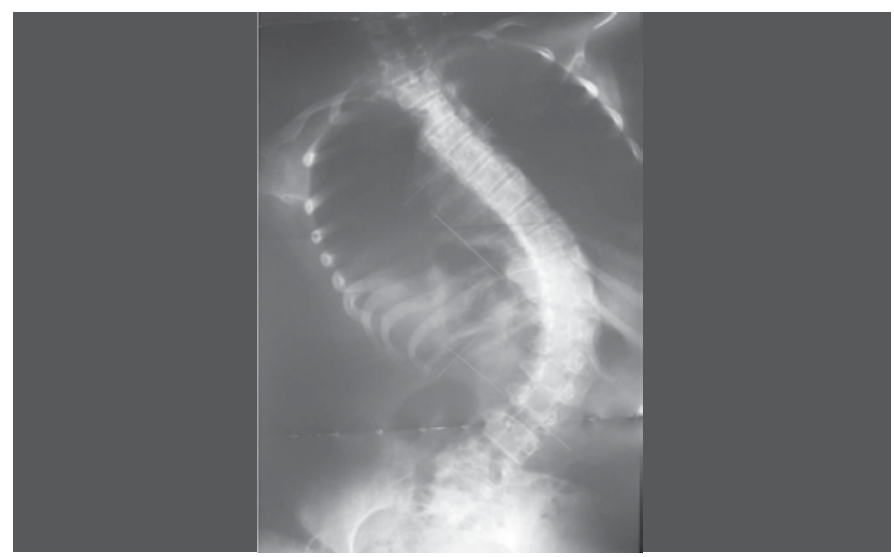

Figura 1. Radiografia AP pré-operatória

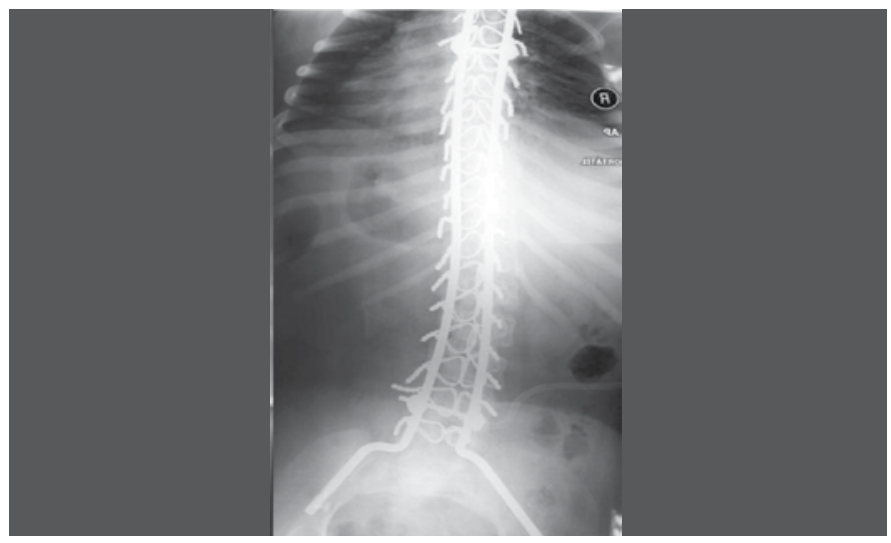

Figura 2. Pós-operatório imediato.

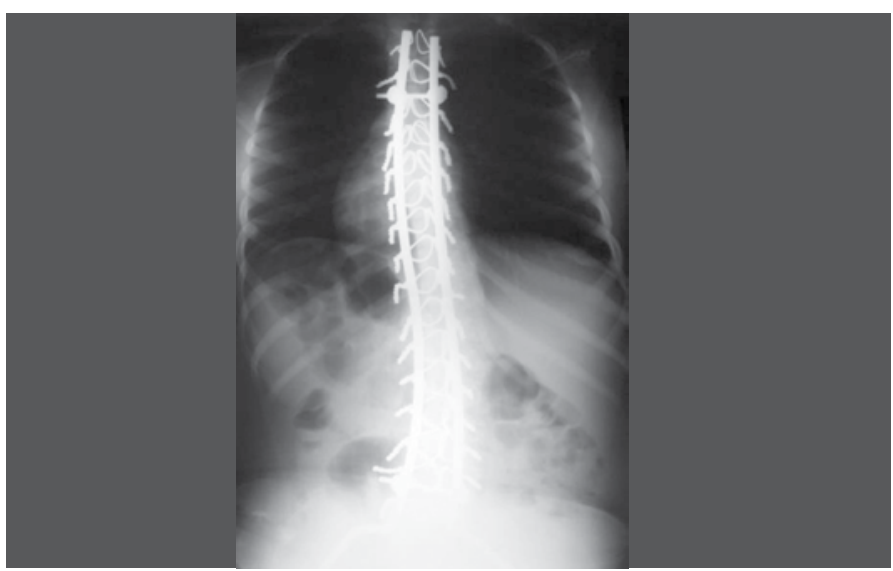

Figura 3. Último seguimento. 


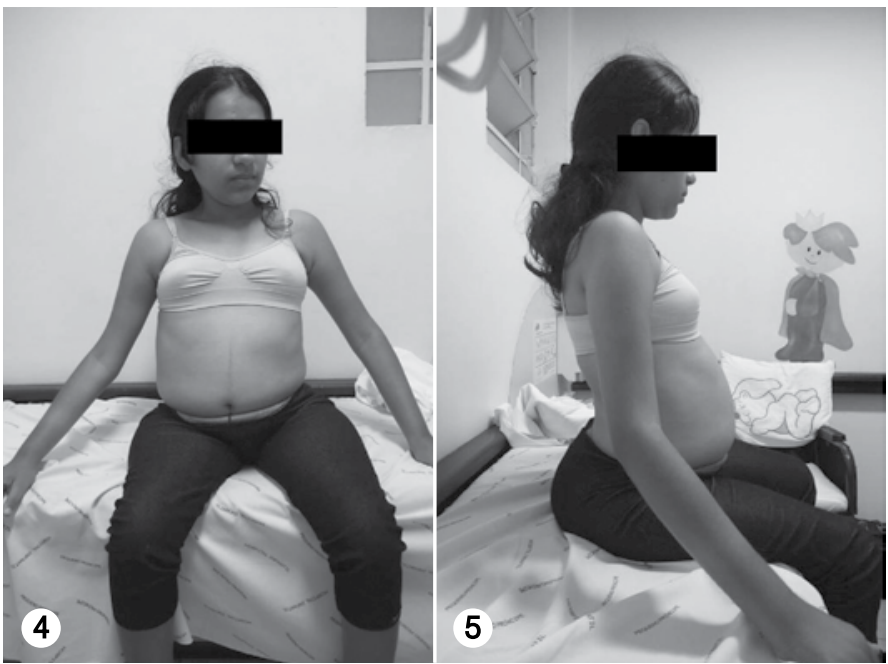

Figuras 4 e 5 - Imagem clínica do paciente no último seguimento.

\section{MATERIAIS E MÉTODOS}

Foi realizada revisão dos prontuários dos pacientes portadores de AME e submetidos ao tratamento cirúrgico de escoliose no Hospital Pequeno Príncipe de 1990 a 2009. Nesse período foram tratados 12 pacientes (Tabela 1 ).

Três pacientes eram do sexo masculino (25\%) e 9 do sexo feminino (75\%). A idade média na data da cirurgia foi 11,21 anos \pm 2,64 anos (variação de 9 a 16 anos) e o peso médio era 25,67 kg \pm $5,36 \mathrm{~kg}$ (variação de 14 a $36 \mathrm{~kg}$ ). Todos eram portadores da forma infantil crônica ou do tipo 2, na escala de função motora FMS tipo I. Quanto a maturidade no momento da cirurgia, aferida pelo sinal de risser, oito pacientes Risser zero $(66,7 \%)$, dois pacientes risser um $(16,7 \%)$, um paciente risser $4(8,3 \%)$ e um paciente risser $5(8,3 \%)$.

Quanto ao padrão da curva, sete pacientes apresentaram curva toracolombar $(58,4 \%)$, três pacientes curva lombar $(25 \%)$ e dois pacientes curva torácica (16,6\%). Os níveis de artrodese variaram quanto à vértebra proximal e distal, sendo cinco pacientes T2-S1 (41,7\%), 3 pacientes T1-S1 (25\%), 2 pacientes T2-L5 (16,7\%), um paciente T1-L5 (8,3\%) e 1 paciente T5-L5 (8,3\%).

A abordagem e instrumentação posterior exclusiva foi utilizada em 9 pacientes (75\%) e abordagem combinada via anterior e posterior em 3 pacientes (25\%). Quanto ao tipo de instrumentação, foi utilizado material de terceira geração em quatro pacientes (33\%), híbrida com amarria e parafuso pedicular em três pacientes (25\%), instrumentação de Hartschill em 3 pacientes (25\%) e amarria sublaminar com fixação no ilíaco pela técnica de Luque-Galveston em três pacientes (25\%)

Em oito pacientes $(66,7 \%)$ foi utilizado enxerto homólogo de banco de tecidos musculoesqueléticos, em três pacientes (25\%) foi utilizado enxerto da crista ilíaca e em um paciente $(8,3 \%)$ foi utilizado enxerto de ilíaco associado à hidroxiapatita.

Foi avaliado o grau de correção e perda da curva principal e da obliqüidade pélvica bem como a capacidade ventilatória no préoperatório e ultimo seguimento, além das complicações cirúrgicas pós-operatórias.

\section{RESULTADOS}

A descrição dos resultados relativos a variáveis quantitativas foi expressa através de médias e desvios padrões. Já para variáveis qualitativas os resultados foram descritos através de frequência e percentual. Para comparação dos resultados de dois momentos consecutivos foi considerado o teste $t$-Student para amostras pareadas.

O seguimento médio foi de 77,5 meses $(6,4$ anos $) \pm 58,9$ meses (4,9 anos).

O ângulo de Cobb coronal médio pré-operatório foi de $76,1^{\circ} \pm$ $31,7^{\circ}$ (variação de $35^{\circ}$ a $144^{\circ}$ ), e pós-operatório de $29,5^{\circ} \pm 23,2^{\circ}$ (variação de $5^{\circ}$ a $90^{\circ}$ ), obtendo correção média de 46,6 $6^{\circ}(61,29 \%)$. $\mathrm{Na}$ ultima avaliação foi de $35,0^{\circ} \pm 24,2^{\circ}$, com uma perda de correção média de $5,5^{\circ}$ (Tabela 2).

A obliquidade pélvica pré-operatória foi de $15,1^{\circ} \pm 13,3^{\circ}$ (variação de $0^{\circ}$ a $37^{\circ}$ ), e no pós-operatório $8,5^{\circ} \pm 9,9^{\circ}$ (variação de $0^{\circ}$ a $30^{\circ}$ ), com uma correção média de $6,5^{\circ}$ (43,37\%). No ultimo seguimento foi de $12,1^{\circ} \pm 9,8^{\circ}$, com uma perda de correção de $3,5^{\circ}$ (Tabela 2)

Cinco pacientes apresentaram complicações pós-operatórias $(41,6 \%)$, sendo que alguns pacientes apresentaram mais de uma complicação. Ocorreram dois casos de infecção (16,6\%), uma no pós-operatório imediato que necessitou desbridamento e curativos, sendo realizado fechamento em $2^{\circ}$ tempo, neste caso foi a segunda cirurgia, porque a primeira realizada em outro serviço a instrumentação foi curta e estava descompensada, ao final de quatro anos de pós-operatório estava bem compensada sem perda da correção obtida e não apresentou recidiva do quadro infeccioso. O outro caso de infecção foi tardia de baixa virulência, com mais de quatro anos de seguimento, necessitou de retirada do implante apresentado

Tabela 1. Perfil dos pacientes operados, acesso utilizado, nível de artrodese, instrumentação e enxerto utilizados, seguimento.

\begin{tabular}{|c|c|c|c|c|c|c|c|c|c|c|}
\hline Paciente & Sexo & Idade & Peso & Risser & Curva & Acesso & Nível & Instrumentação & Enxerto & Seguimento \\
\hline 1 & $\mathrm{~F}$ & $9 a+8 m$ & $26 \mathrm{~kg}$ & Zero & TLD & VP & T2-S1 & Hartschill & lliaco + Hidroxiapatita & $5 a+2 m(62 m)$ \\
\hline 2 & $\mathrm{~F}$ & $9 a+5 m$ & $24 \mathrm{~kg}$ & Zero & TLE & $V A+V P$ & T2-S1 & Luque-Galveston & lliaco & $6 a+10 m(82 m)$ \\
\hline 3 & $\mathrm{~F}$ & $8 a+9 m$ & $21 \mathrm{~kg}$ & Zero & $T L D$ & $V A+V P$ & $\mathrm{~T} 2-\mathrm{S} 1$ & Hartschill & Aloenxerto & Óbito \\
\hline 4 & $\mathrm{~F}$ & $8 a+4 m$ & $28 \mathrm{~kg}$ & Zero & TD & $V A+V P$ & T2-L5 & Híbrido & Aloenxerto & $9 a+9 m(117 m)$ \\
\hline 5 & $\mathrm{~F}$ & $10 a+1 m$ & $25 \mathrm{~kg}$ & Zero & TLD & VP & T1-L5 & Parafusos Pediculares & Aloenxerto & $2 a(24 m)$ \\
\hline 6 & M & $10 a+2 m$ & $28 \mathrm{~kg}$ & Zero & TLD & VP & $\mathrm{T} 2-\mathrm{S} 1$ & Hibrido & Aloenxerto & $5 a+10 m(70 m)$ \\
\hline 7 & $\mathrm{~F}$ & $12 a+6 m$ & $26 \mathrm{~kg}$ & 1 & LD & VP & T1-S1 & Luque-Galveston & Aloenxerto & $2 a(24 m)$ \\
\hline 8 & $M$ & $11 a+3 m$ & $28 \mathrm{~kg}$ & Zero & LD & VP & $\mathrm{T} 1-\mathrm{S} 1$ & Hartschill & lliaco & $19+9 m(237 m)$ \\
\hline 9 & F & $15 a+8 m$ & $36 \mathrm{~kg}$ & 4 & LD & VP & $\mathrm{T} 2-\mathrm{S} 1$ & Hibrido & lliaco & $5 a+6 m(66 m)$ \\
\hline 10 & $M$ & $9 a+2 m$ & $14 \mathrm{~kg}$ & Zero & TLE & VP & T5-L5 & Parafusos Pediculares & Aloenxerto & $5 a+9 m(69 m)$ \\
\hline 11 & $\mathrm{~F}$ & $13 a+5 m$ & $22 \mathrm{~kg}$ & 1 & TLD & VP & T1-S1 & Parafusos Pediculares & Aloenxerto & $4 a+1 m(49 m)$ \\
\hline 12 & $\mathrm{~F}$ & $16 a+1 m$ & $30 \mathrm{~kg}$ & 5 & TE & VP & T2-L5 & Parafusos Pediculares & Aloenxerto & $4 a+5 m(53 m)$ \\
\hline
\end{tabular}


a resolução do quadro. Na retirada do implante foi identificado pseudartrose na transição toracolombar que manteve-se assintomática, sem perda da correção com 2 anos de pós-operatório. Um paciente apresentou progressão da curva proximal ao implante com necessidade de estender da artrodese. Um paciente apresentou quebra do material, sem necessidade de intervenção. Um paciente evoluiu com óbito $(8,3 \%)$ no pós-operatório imediato, secundário a insuficiência respiratória aguda (Tabela 3).

Tabela 2. Correção do ângulo de Cobb e obliquidade pélvica.

\begin{tabular}{c|c|c|c|c|c|c}
\hline Paciente & Pré-op & $\begin{array}{c}\text { Cobb } \\
\text { PO im }\end{array}$ & Último & Pré-op & $\begin{array}{c}\text { Obliquidade PO } \\
\text { imediato }\end{array}$ & Último \\
\hline 1 & $35^{\circ}$ & $24^{\circ}$ & $30^{\circ}$ & $3^{\circ}$ & $0^{\circ}$ & $5^{\circ}$ \\
\hline 2 & $86^{\circ}$ & $42^{\circ}$ & $58^{\circ}$ & $37^{\circ}$ & $20^{\circ}$ & $27^{\circ}$ \\
\hline 3 & $70^{\circ}$ & $5^{\circ}$ & $5^{\circ}$ & $0^{\circ}$ & $0^{\circ}$ & $20^{\circ}$ \\
\hline 4 & $115^{\circ}$ & $30^{\circ}$ & $35^{\circ}$ & $0^{\circ}$ & $1^{\circ}$ & $2^{\circ}$ \\
\hline 5 & $56^{\circ}$ & $5^{\circ}$ & $8^{\circ}$ & $16^{\circ}$ & $3^{\circ}$ & $5^{\circ}$ \\
\hline 6 & $90^{\circ}$ & $16^{\circ}$ & $16^{\circ}$ & $26^{\circ}$ & $12^{\circ}$ & $12^{\circ}$ \\
\hline 7 & $48^{\circ}$ & $18^{\circ}$ & $33^{\circ}$ & $12^{\circ}$ & $13^{\circ}$ & $11^{\circ}$ \\
\hline 9 & $76^{\circ}$ & $34^{\circ}$ & $50^{\circ}$ & $17^{\circ}$ & $3^{\circ}$ & $11^{\circ}$ \\
\hline 10 & $65^{\circ}$ & $30^{\circ}$ & $30^{\circ}$ & $25^{\circ}$ & $12^{\circ}$ & $10^{\circ}$ \\
\hline 11 & $52^{\circ}$ & $34^{\circ}$ & $90^{\circ}$ & $30^{\circ}$ & $30^{\circ}$ & $30^{\circ}$ \\
\hline
\end{tabular}

Tabela 3. Complicações.

\begin{tabular}{c|c}
\hline Paciente & Complicações \\
\hline 1 & $n$ \\
\hline 2 & $n$ \\
\hline 3 & Obito \\
\hline 4 & $n$ \\
\hline 5 & $n$ \\
\hline 6 & $n$ \\
\hline 7 & $n$ \\
\hline 8 & Infeção, Pseudartrose \\
\hline 9 & Alongamento da artrodese, Óbito \\
\hline 10 & Quebra de material \\
\hline 11 & Infecção \\
\hline 12 &
\end{tabular}

A função pulmonar foi avaliada através do percentual obtido/ preditivo da capacidade vital forcada (CVF) no pré-operatório e no ultimo seguimento. Foram excluídos da análise os dois pacientes que apresentaram óbito e um paciente que não realizou espirometria no pós-operatório. Ao total 9 pacientes (75\%) apresentaram CVF media de $62,9 \% \pm 38,6 \%$ (variação de 23,3\% a 89\%) no préoperatório. No ultimo seguimento a CVF media foi de $45,9 \% \pm$ $25,0 \%$ (variação de 15\% a 86,2\%), apresentando um declínio de $17 \%$ da capacidade vital, diminuição de $2,4 \%$ por ano de seguimento. Apesar da média apresentar declínio, 3 pacientes apresentaram melhora da CVF (Tabela 4).

Dois pacientes evoluíram com óbito, um no pós-operatório imediato devido à insuficiência respiratória aguda, sendo excluído da análise estatística, e outro com 5 anos e 9 meses de seguimento.

\section{DISCUSSÃO}

O objetivo do tratamento cirúrgico da deformidade da coluna vertebral no pacientes portador de AME visa a melhora da qualidade de vida obtendo a liberação das mãos para realização das atividades diárias, permite melhora da condição de frequentar a escola, a melhora da auto- imagem, bem como minimiza o impacto negativo da deformidade da coluna na função pulmonar ${ }^{9}$. Granata et al. ${ }^{4}$ relataram que os pacientes não tratados apresentaram grande prejuízo estético e deterioração da função, obrigando-os a utilizar os membros superiores para manter o equilíbrio sentado.

A maioria das series mostra um percentual de correção da escoliose que varia de 40 a $50 \%^{10-13}$. Nossa amostra a correção da curva foi de $41^{\circ}(61 \%)$ com uma perda de $5^{\circ}$ no último seguimento. A correção da obliqüidade pélvica foi de $6,5^{\circ}$, semelhante as encontradas na literatura ${ }^{10,13}$. Em comparação a outros estudos a perda da correção na ultima avalição foi maior, no entanto este estudo é o que tem o tempo maior de seguimento médio que foi de 6,4 anos.

Pacientes portadores de escoliose neuromuscular submetidos ao tratamento cirúrgico estão sujeitos a complicação no pós-operatório. De acordo com diversos autores varia $24-75 \%{ }^{6}$ Mohamad et al. ${ }^{6}$ relataram aproximadamente $33,1 \%$ e que as complicações mais frequentes acometem o sistema respiratório (19,4\%). Neste estudo encontramos complicações pós-operatórias em $41,6 \%$ dos casos, sendo que em sua maioria foram resolvidas e não trouxeram prejuízo final ao resultado do tratamento.

A deterioração da função pulmonar é condição inerente aos portadores de AME e escoliose ${ }^{5}$. Os dados encontrados na literatura apontam para a continuidade do declínio da função pulmonar após o tratamento cirúrgico, porém em menor velocidade que no pré-operatório e os não tratados cirurgicamente. Somente Robinson et al. ${ }^{7}$ encontraram melhora da CVF após a correção cirúrgica.

A longo prazo o declínio da função pulmonar parece ocorrer

TABELA 4. Variação da CVF no pré-operatório e no último seguimento.

Capacidade Ventilatória Forçada (CVF)

\begin{tabular}{|c|c|c|c|c|c|c|}
\hline \multicolumn{4}{|c|}{ Pré-operatória } & \multicolumn{3}{|c|}{ Último Seguimento } \\
\hline Paciente & Preditivo & Obtido & $\%$ do previsto & Preditivo & Obtido & $\%$ do previsto \\
\hline 1 & 2,06 & 0,8 & $39 \%$ & 2,59 & 0,72 & $27 \%$ \\
\hline 2 & 1,37 & 0,59 & $43 \%$ & 2,88 & 0,56 & $19 \%$ \\
\hline 3 & 2,4 & 0,56 & $23 \%$ & 2,74 & 0,88 & $32 \%$ \\
\hline 4 & 2,15 & 1,21 & $56 \%$ & 3,1 & 0,47 & $15 \%$ \\
\hline 5 & 1,74 & 2,54 & $146 \%$ & 1,86 & 1,6 & $86 \%$ \\
\hline 6 & 2 & 1,53 & $77 \%$ & 4,7 & 2,86 & $60 \%$ \\
\hline 7 & 1,89 & 1,68 & $89 \%$ & 2,52 & 1,58 & $62 \%$ \\
\hline 8 & 2,01 & 0,48 & $23 \%$ & 2,94 & 0,95 & $32 \%$ \\
\hline 9 & 3,47 & 2,38 & $68 \%$ & 3,75 & 2,9 & $77 \%$ \\
\hline
\end{tabular}


mais lentamente. Agudamente após a cirurgia existe tendência a uma redução acentuada da capacidade pulmonar, devido a modificação e acomodação da caixa torácica. Chong et al. ${ }^{10}$, em um estudo comparativo do tratamento cirúrgico da escoliose em pacientes com Distrofia Muscular de Duchenne (DMD) e AME, encontraram piora de $10 \%$ da capacidade vital no $1^{\circ}$ ano após o tratamento cirúrgico de pacientes com DMD e AME, com estabilização do declínio no segundo ano em cerca de 3\% no grupo AME e $2 \%$ no grupo DMD. Chng et al. ${ }^{8}$ relataram redução da CVF/ano de $7,7 \%$ para $3,8 \%$ em 3,6 anos de seguimento pós-operatório através espirometrias seriadas. Neste estudo, encontramos redução média de 17\% da CVF em um seguimento médio de 6,4 anos, contudo, 3 pacientes apresentaram melhora da capacidade pulmonar no pós-operatório. Nos estudos em que houve analise clinica dos pacientes, a maioria das famílias perceberam uma melhora global e da função pulmonar em relação a complicações, como pneumonias ${ }^{9,14}$. Esta coleta dos dados especifica não foi realizada em nossos pacientes, porém nenhum apresentou novo quadro pulmonar grave ou que necessitasse de internamento e tinham histórico prévio de deterioração no pré-operatório. Os resultados encontrados neste estudo com seguimento médio de 6,4 anos e os da literatura sugerem o benefício do tratamento cirúrgico da escoliose no que tange a deterioração da função pulmonar, contudo estudos prospectivos com uma amostra maior e seguimento mais longo são necessários para conclusões definitivas.

O tratamento cirúrgico da escoliose proporcionou diversos benefícios através correção e estabilização da deformidade. As complicações não fatais, foram resolvidas sem prejuízo no resultado final do tratamento.

\section{REFERÊNCIAS}

1. Dubowitz $V$. Disorders of the lower motor neurone: the spinal muscular atrophies. In: Dubowitz V, editor. Muscle disorders in childhood. 2nd ed. London: Saunders;1995. p.325-69.

2. Merlini L, Granata C, Bonfiglioli S, Marini ML, Cervellati S, Savini R.Scoliosis in spinal muscular atrophy: natural history and management. Dev Med Child Neurol. 1989;31(4):501-8

3. Bowen JR, Forlin E. Spinal muscular atrophy. In: Weinstein SL editor. The pediatric spine: principles and practice. New York: Raven Press; 1994. p.1025-40.

4. Granata C, Merlini L, Magni E, Marini ML, Stagni SB. Spinal muscular atrophy: natura history and orthopaedic treatment of scoliosis. Spine (Phila Pa 1976).1989;14(7):760-2.

5. Sucato DJ. Spine deformity in spinal muscular atrophy. J Bone Joint Surg Am.2007;89 (Suppl 1):148-54.

6. Mohamad F, Parent S, Pawelek J, Marks M, Bastrom T, Faro F, et al. Perioperative complications after surgical correction in neuromuscular scoliosis. J Pediatr Orthop. 2007;27(4):392-7.

7. Robinson D, Galasko CS, Delaney C, Williamson JB, Barrie JL. Scoliosis and lung function in spinal muscular atrophy. Eur Spine J. 1995;4(5):268-73.

8. Chng SY, Wong YQ, Hui JH, Wong HK, Ong HT, Goh DY. Pulmonary function and scoliosis in children with spinal muscular atrophy types II and III. J Paediatr Child Health. 2003;39(9):673-6.
9. Bridwell KH, Baldus C, Iffrig TM, Lenke LG, Blanke K. Process measures and patient/ parent evaluation of surgical management of spinal deformities in patients with progressive flaccid neuromuscular scoliosis (Duchenne's muscular dystrophy and spinal muscular atrophy). Spine (Phila Pa 1976). 1999:24(13):1300-9.

10. Chong HS, Moon ES, Kim HS, Ankur N, Park JO, Kim JY et al. Comparison between Operated Muscular Dystrophy and Spinal Muscular Atrophy Patients in terms of Radiological, Pulmonary and Functional Outcomes. Asian Spine J. 2010:4(2):82-8

11. Bentley G, Haddad F, Bull TM, Seingry D. The treatment of scoliosis in muscular dystrophy using modified Luque and Harrington-Luque instrumentation. J Bone Joint Surg Br. 2001:83(1):22-8.

12. Harrington PR. Treatment of scoliosis. Correction and internal fixation by spine instrumentation. J Bone Joint Surg Am. 1962;44:591-610.

13. Santos DC, Cavali PTM, Santos MAM, Lehoczki MA, Rossato AJ, Landim E. Tratamento cirúrgico da escoliose em pacientes com amiotrofia espinhal com parafusos pediculares (instrumental de $3^{\mathrm{a}}$ geração) e complicações precoces. Coluna/Columna. 2010;9(2):98-103.

14. 14. Aprin H, Bowen JR, MacEwen GD, Hall JE. Spine fusion in patients with spinal muscular atrophy. J Bone Joint Surg Am. 1982;64(8):1179-87. 